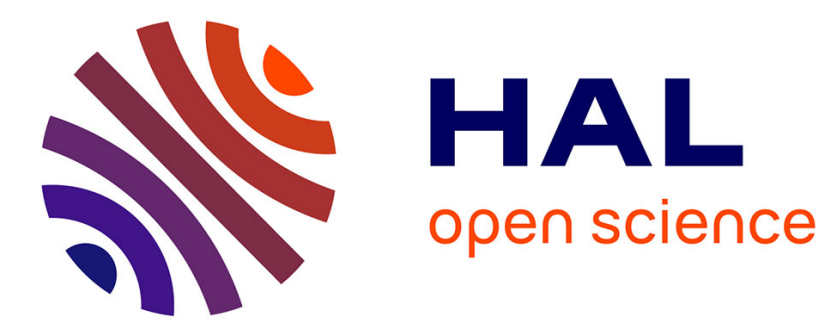

\title{
Characterization of multilayer coatings by X-ray reflection
}

\author{
E. Spiller
}

\section{To cite this version:}

E. Spiller. Characterization of multilayer coatings by X-ray reflection. Revue de Physique Appliquée, 1988, 23 (10), pp.1687-1700. 10.1051/rphysap:0198800230100168700 . jpa-00245997

\section{HAL Id: jpa-00245997 https://hal.science/jpa-00245997}

Submitted on 1 Jan 1988

HAL is a multi-disciplinary open access archive for the deposit and dissemination of scientific research documents, whether they are published or not. The documents may come from teaching and research institutions in France or abroad, or from public or private research centers.
L'archive ouverte pluridisciplinaire HAL, est destinée au dépôt et à la diffusion de documents scientifiques de niveau recherche, publiés ou non, émanant des établissements d'enseignement et de recherche français ou étrangers, des laboratoires publics ou privés. 
Classification

Physics Abstracts

$42.78 \mathrm{H}-61.10-78.65$

\title{
Characterization of multilayer coatings by X-ray reflection
}

\author{
E. Spiller \\ IBM Research Division, T. J. Watson Research Center, Box 218, Yorktown Heights, N.Y. 10598, U.S.A.
}

(Reçu le $1^{\text {er }}$ décembre 1987, révisé le 19 février 1988, accepté le 10 mars 1988)

\begin{abstract}
Résumé. - On présente les méthodes permettant de déduire les caractéristiques d'un système multicouches à partir d'une courbe de réflectivité. Dans le cas d'empilements comportant peu de couches, les méthodes d'inversion permettent de déduire l'épaisseur de chaque couche et les rugosités de chaque interface. Si le système est composé de beaucoup de couches, une description statistique de l'empilement conduit à des formules simples fournissant une moyenne des erreurs sur la période et sur les rugosités des interfaces d'un miroir multicouches.
\end{abstract}

\begin{abstract}
Methods to deduce the parameters of a multilayer X-ray mirror from its reflectivity curve are reviewed. Inversion methods are successful for coatings with few layers and give the thickness of each layer and the roughness of each interface. A statistical description of the coating is used for coatings with many layers, and simple formulas are given to derive the average thickness error and boundary roughness in a multilayer mirror.
\end{abstract}

\section{Introduction.}

The calculation of the performance of a multilayer mirror from its structure poses no problem, and a rigorous solution can easily be obtained in a short time on any microcomputer. The inverse problem, the determination of the multilayer parameters from a measured reflectivity curve has for the general case no unique solution. This is mainly due to the fact that only the intensity but not the phase of the reflected wave is measured. Furthermore, in a coating with absorbing layers, the bottom layers often contribute very little to the reflectivity and may be reconstructed only with large uncertainty. The chances that the structure obtained by the inversion process is close to the real structure can be increased by decreasing the number of free parameters in the multilayer and increasing the number of data points in the measurements. Coatings with only few layers can be reconstructed with large confidence. For a coating with a large number of layers, the number of free parameters can be reduced by using a statistical description of the mutilayer. An average thickness error in a nearly periodic coating can be obtained with a much higher level of confidence than all the individual thicknesses.

The derivation of a structure from measurements REVUE DE PHYSIQUE APPLIQUÉE. - T. 23, N` 10, OCTOBRE 1988 often starts with a definition of a merit function, whose value increases with increasing differences between measured and calculated intensities, and a starting guess of the structure. The speed of convergence and the reliability of the result depend on the choice of the merit function, on the initial guess, and on good intuition. An understanding of which possible structures might produce what features is very helpful in this selection. Nearly always a good intuition produces better results than a formal computer optimization from an arbitrary starting point. The computer optimization is most useful for a final refinement from an intuitively found structure that is already close to the correct values. The main purpose of this paper is to develop this intuition. We will concentrate on structures with few free parameters and use the statistical description for coatings with many layers. This allows us in most cases to derive the parameters of a coating from simple formulas and an inspection of the measurements.

The motivation for the work described in this paper was the desire to characterize a coating in such a way that the necessary improvements for fabrication of coatings with better performance could be obtained from this characterization. The performance of a coating is mostly limited by two parameters, the quality of the interfaces, and the 
thickness errors of the layers, and it is important to determine these parameters separately.

\section{Multilayer theory.}

Calculation of the reflectivity and transmission of a multilayer structure is straightforward and treated in any text-book on optical thin films [1]. One can use either matrix methods (each film or each boundary is characterized by a matrix and a multilayer by the product matrix) or calculate the multilayer performance by the recursive use of the single film formula. These theories are equivalent to Ewald's dynamical theory of X-ray diffraction [2]. As a reference for our discussion we give here the formulas for the recursive method. One starts with the Fresnel coefficient $r_{12}$ for the reflected amplitude from a single boundary between two materials of complex refractive indices $n_{1}$ and $n_{2}$ and propagation angles $\alpha_{1}, \alpha_{2}$,

$$
\begin{aligned}
& r_{12}^{\mathrm{s}}=\frac{n_{1} \cos \alpha_{1}-n_{2} \cos \alpha_{2}}{n_{1} \cos \alpha_{1}+n_{2} \cos \alpha_{2}}, \text { s-polarization } \\
& r_{12}^{\mathrm{p}}=\frac{n_{1} \cos \alpha_{2}-n_{2} \cos \alpha_{1}}{n_{1} \cos \alpha_{2}+n_{2} \cos \alpha_{1}}, \text { p-polarization }
\end{aligned}
$$

where the propagation angles $\alpha$ are calculated from the propagation angle $\alpha_{0}$ in the incident medium (index $n_{0}$ ) using Snell's law :

$$
\begin{gathered}
n \sin \alpha=n_{0} \sin \alpha_{0}, \\
\cos \alpha=\sqrt{1-\left(n_{0} / n\right)^{2} \sin ^{2} \alpha_{0}} .
\end{gathered}
$$

In the soft X-ray region, the refractive indices of all materials (except vacuum) are complex leading also to complex values of the propagation angle in equations (2). The incident angle $\alpha$ is measured from the normal and can be replaced by the grazing angle $\theta=90-\alpha$.

The refractive index $n$ of a material can be expressed by the atomic scattering factor [3] $f=f_{1}+i f_{2}$ with

$$
n=1-\delta+i k=1-\frac{r_{0} \lambda^{2}}{2 \pi} N_{\text {at }}\left(f_{1}-i f_{2}\right) .
$$

Here $r_{0}=2.82 \times 10^{-13} \mathrm{~cm}$ is the classical electron radius and $N_{\text {at }}$ is the number of atoms of a specific type per $\mathrm{cm}^{3}$ of material. For a compound the index is obtained by a summation of equation (3) for the contributions from all its elements. Interaction between atoms which might influence the electronic structure in a solid are neglected in equation (3).

The reflected amplitude of a single film of thickness $d$ with two boundaries (top and bottom) with amplitude reflectivity $r_{\mathrm{t}}, r_{\mathrm{b}}$ and amplitude transmission of the top boundary $t_{\mathrm{t}}^{+}, t_{\mathrm{t}}^{-}$is given by

$$
r_{\mathrm{f}}=r_{\mathrm{t}}+\frac{r_{\mathrm{b}} t_{\mathrm{t}}^{+} t_{\mathrm{t}}^{-} \exp (2 i \varphi)}{1+r_{\mathrm{t}} r_{\mathrm{b}} \exp (2 i \varphi)},
$$

with

$$
\begin{gathered}
\varphi=\frac{2 \pi}{\lambda} n d \cos \alpha, \\
\varphi=\frac{2 \pi}{\lambda} d \sqrt{n^{2}-n_{0}^{2} \sin ^{2} \alpha_{0}} .
\end{gathered}
$$

The amplitude transmission $t^{+}=t_{12}$ from medium 1 to 2 for s- and p-polarization is given by

$$
\begin{aligned}
& t_{12}^{\mathrm{s}}=\frac{2 n_{1} \cos \alpha_{1}}{n_{1} \cos \alpha_{1}+n_{2} \cos \alpha_{2}}, \\
& t_{\mathrm{P}}=\frac{2 n_{1} \cos \alpha_{1}}{n_{2} \cos \alpha_{1}+n_{1} \cos \alpha_{2}},
\end{aligned}
$$

and the corresponding value $t^{-}=t_{21}$, by exchange of the indices 1 and 2 in equations (6).

One can easily verify from the Fresnel equation that

$$
t_{12} t_{21}+r_{12}^{2}=1
$$

and use this expression to rewrite equation (4) to obtain

$$
r_{\mathrm{f}}=\frac{r_{\mathrm{t}}+r_{\mathrm{b}} \exp (2 i \varphi)}{1+r_{\mathrm{t}} r_{\mathrm{b}} \exp (2 i \varphi)} .
$$

Equations (4) and (8) are valid for any thin film, even if it is bounded on either side by multiple layers. Recursive application of equation (8) gives the reflectivity of any coating, for example by starting with $r_{\mathrm{b}}$ using the Fresnel coefficient between the first film and the substrate and then using the calculated value of $r_{\mathrm{f}}$ as a new value for $r_{\mathrm{b}}$ when the next film is added.

We will in this paper analyse reflectivity curves obtained at short wave-lengths (around $\lambda=1 \AA$ ) and for small grazing angles. Polarization effect can be neglected in this case and the formulas for spolarization are sufficient for all calculations.

In X-ray diffraction it is convenient to introduce momentum vectors in reciprocal space to analyse a pattern. In the one-dimensional multilayer system, where only specular reflection at planes parallel to the substrate is measured, only the $z$-component is relevant. We introduce this component as

$$
q=\frac{4 \pi}{\lambda} n \cos \alpha=\frac{4 \pi}{\lambda} n \sin \theta,
$$

and can express all formulas as function of $q$. We obtain for the Fresnel equation

$$
r_{12}=\frac{q_{1}-q_{2}}{q_{1}+q_{2}} .
$$


$q$ represents the change in momentum of a photon at specular reflection. The introduction of $q$ simplifies the analysis of a structure in the $X$-ray region, because the 2 variables $\lambda$ and $\theta$ are replaced by the single variable $q$. The imaginary part of $q$ can often be neglected at short wave-lengths.

KINEMATICAL THEORY (VECTOR MODEL) AND FOURIER TRANSFORM. - Multiple reflections and the depletion of the incident beam can be neglected if the reflectivity at each boundary and the total reflectivity of the structure are small. In this approximation the reflectivity is simply calculated as the vector sum of the reflected amplitudes of the individual boundaries :

$$
r=\sum_{j=0}^{N} r_{j} \mathrm{e}^{2 i \varphi_{j}},
$$

where $\varphi_{j}$ is now the phase retardation of the $j$ th boundary measured from the top surface $r_{0}$. Expressed in $q$ this yields the Fourier series

$$
r=\sum_{j=0}^{N} r_{j}\left(Z_{j}\right) \mathrm{e}^{i q z_{j}},
$$

where $z$ is the depth coordinate in the multilayer and $r_{j}$ is the reflected amplitude of the boundary at coordinate $z_{j}$.

For the case that $r_{j}$ is a continuous function $r(z)$ equation (12) becomes a Fourier integral

$$
r(q)=\int_{0}^{\infty} r(z) \mathrm{e}^{i q z} \mathrm{~d} z
$$

with the inversion

$$
r(z)=\frac{1}{2 \pi} \int_{0}^{\infty} r(q) \mathrm{e}^{-i q z} \mathrm{~d} q .
$$

The calculation of a multilayer reflectivity by Fourier transformation takes considerably less computer time than the full theory. The multilayer structure is treated like a diffraction grating, where all lines contribute with equal weight to the diffracted beam. It is also possible to approximate the depletion of the incident beam in the multilayer structure by multiplying $r(z)$ in equation (12) or (13) with a weighting factor $\mathrm{e}^{-z / z_{\max }}$, where $z_{\max }$ represents an estimate of the penetration length of the radiation into the multilayer. The inverse Fourier relation equation (14) is useful as a first step in the design of a multilayer with a desired reflectivity curve $r(q)$. The main difficulty to derive the structure of a multilayer from a measured reflectivity curve $r(q)$ by Fourier transform is the fact that usually only the amplitude but not the phase of $r(q)$ is measured.

Figure 1 compares the results obtained with the exact theory to those obtained by the vector model for a periodic multilayer mirror with 64 periods. The curves for the vector model on the right are obtained from equation (12), where the reflected amplitude at each boundary is approximated by

$$
\begin{gathered}
r= \pm \frac{\left(\delta_{1}-\delta_{2}\right)+i\left(k_{1}-k_{2}\right)}{2 \sin ^{2} \theta} \mathrm{e}^{-z / z_{\max }} \\
z_{\max }=\frac{1.58 \lambda}{2 \pi\left(k_{1}+k_{2}\right) \sin \theta} .
\end{gathered}
$$
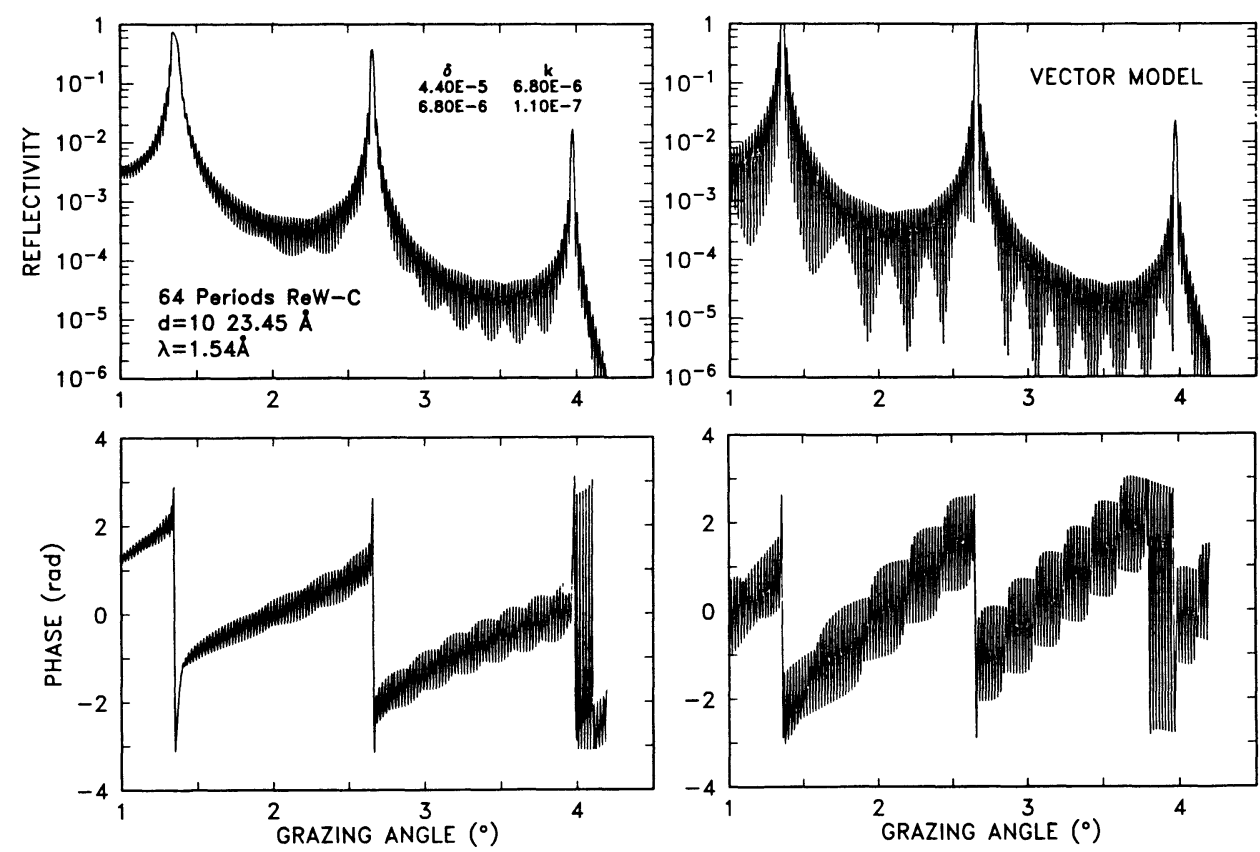

Fig. 1. - Reflectivity and phase shift on reflection at $\lambda=1.54 \AA$ for a 128 layer periodic ReW-C multilayers mirror calculated with the exact theory (left) and the vector model or kinematical theory (right). The oscillations in the envelope of the minima are artifacts produced by the choice of the sampling points. 
The exponential in equation (15a) describes the smaller contribution of the boundaries deeper in the structure and the first factor is an approximation to the Fresnel coefficient. The alternating sign in equation (15a) describes the phase shift of $180^{\circ}$ between adjacent boundaries.

Figure 1 demonstrates that the vector model is in good agreement with the correct theory whenever the reflectivity is small. The largest errors occur around the main reflectivity maximum, where the vector model produces reflectivities above $100 \%$. Here the incident beam is mostly depleted by reflection rather than absorption, and such depletion is not included in our model calculation. One notices also that the oscillations between the main peaks are too large. These oscillations are produced by interference between the top and bottom boundary of the multilayer stack and have the largest contrast when both boundaries contribute with equal weight. We can reduce the amplitude of this oscillation by choosing a smaller value for $z_{\max }$ than equation (15b) which results in a smaller contribution from the bottom boundary. We conclude that the vector model is useful for the characterization of a multilayer system at large grazing angles and high orders, where reflectivities are low. Due to its simplicity, and because it describes also the performance of the simple diffraction grating, the vector model is very helpful for developing an intuition about the expected performance of a multilayer system.

IMPERFECT BOUNDARIES. - The Fourier transformation (Eq. (13)) can be used to calculate the reduction of the reflectivity of a boundary by roughness or by a gradual transition between two materials. Describing the boundary by a Gaussian distribution of the reflected amplitude around $z=0$,

$$
r(z)=\frac{r_{0}}{\sigma \sqrt{2 \pi}} \exp -\left(\frac{z^{2}}{2 \sigma^{2}}\right),
$$

we obtain from the Fourier integral between $-\infty$ and $\infty$

$$
r(q)=r_{0} \mathrm{e}^{-\frac{q^{2} \sigma^{2}}{2}},
$$

where $r_{0}$ is the amplitude reflectivity of a perfectly smooth, abrupt boundary $(\sigma=0)$.

The reduction of the reflected intensity is given by

$$
R(q)=R_{0} \mathrm{e}^{-q^{2} \sigma^{2}},
$$

i.e. the reflection loss has the well known DebyeWaller form.

We can easily incorporate imperfect boundaries into the multilayer calculations by multiplying each
Fresnel coefficient for the amplitude with the corresponding Debye-Waller factor (Eq. (17)) before insertion into equation (4) or (8) [4].

By using equation (8) for the calculation, we assume that equation (7) is valid, i.e. the boundary is lossless. This is a good approximation for smooth transition layers of width $\sigma$ without any scattering losses. The transition layer reduced the reflectivity but increases the transmission by an equivalent amount. Absorption losses due to propagation of radiation through the transition layer are not explicity included in this treatment, because we do not subtract the thickness of the transition layer from the layer thickness in our model. Therefore these losses are already represented by the losses in the adjacent films. A model of the surface and a theory which describes the scattering distribution from the boundary is required to derive the modified transmission coefficient (Eq. (6)) for rough surfaces. Such a theory could derive an efficiency value $\eta(\eta<1)$ for each boundary and this value would replace the value 1 on the right side of equation (7) and appear as a factor in the second term of the nominator in equation (8). The differences in the performance between multilayer mirrors with scattering or with smooth boundaries are often smaller than the experimental error and it is necessary to measure the scattered light to distinguish the two cases [4].

The Debye-Waller factor was obtained by Fourier transformation from the distribution function of the reflectivity around a boundary and a Gaussian distribution was assumed to derive equation (18). Other distribution functions produce a different Debye-Waller factor. A measurement of $R(q) / R_{0}(q)$ over a large range of $q$ values can be used to determine the shape of the distribution function. However, the author is not aware of published measurements where a non-Gaussian distribution was used to fit the data. The performance of a coating with transition layers can of course always be calculated by replacing the transition layer with a large number of homogeneous films with the desired distribution of the optical constants. The use of a Debye-Waller type factor as described above requires less computer time and gives practically the same result for multilayer mirrors in the soft X-ray and X-ray region.

\section{Inversion of multilayer calculation.}

The inversion problem occurs in many branches of physics and many sophisticated methods have been developed. Examples are the structure determination from an X-ray diffraction pattern, reconstruction of objects from holographic or tomographic recordings, geological explorations by means of acoustic (seismic) waves, design of coatings with a desired performance and many more. A unique 
reconstruction can only be obtained if amplitude as well as phase are determined. In all cases the set of experimental data should be considerably larger than the number of free parameters in the inversion process.

For the characterization of a multilayer coating reflectivity measurements at short wavelengths in the X-ray region are preferred over measurements at soft X-rays, because they offer the potential to obtain data over a larger $q$ range (Eq. (9)) and are less affected by absorption. In addition, the optical constants are more reliably known at shorter wavelengths. A measurement of the reflectivity $R(\theta)$ for a fixed wavelength is preferred over a measurement of $R(\lambda)$ at fixed angle, because the optical constants do not change for the measurement in the first case resulting in a smaller number of free parameters.

The inversion problem is reduced to a 1-dimensional problem if we consider only the specular reflection from a multilayer (i.e. the normal component of the momentum vector is reversed, the other components remain constant). The reflectivity is determined by the distribution of matter in the $z$ direction normal to the surface; variations in the other coordinates are averaged out, and in the inversion process only the distribution of matter in this direction can be obtained.

Possible inversion methods are classified in table I. One usually starts any analysis with method (1). For systems with few layers this method works very well and we will in the following develop the intuition required for the initial guess. The search for further improvement after the first step can be automated with method (2). The choice of the merit function and the quality of the initial guess determine the speed of convergence and the quality of the final result. The method can converge to a wrong solution! The statistical characterization (3) is appropriate to derive average thickness errors and bound-

Table I. - Inversion methods.

\section{Model calculation}

- Guess Structure and calculate reflectivity

- Compare with data

- Improve initial guess and repeat

2. Numerical minimization

- Define merit function

- Minimize merit function numerically

3. Statistical Characterization

- Derive thickness error and roughness from measured data

4. Direct methods

- Estimate phases from amplitude

- Use probability or analytic continuation

- Invert calculation, inverse Fourier approximation ary roughness, but does not give individual parameters of a multilayer system. The averages can be derived from the measured data using simple formulas. Direct methods [5] have become important for structure analysis by X-ray diffraction. One tries to invert the problem directly without the use of a specific model. The phase has to be estimated from the measured amplitude, and one tries to use probability arguments, $a$ priori knowledge, and/or dispersion relations for this estimate. The inverse Fourier transform can be used for an approximate inversion.

\section{MODEl CALCULATION.}

Single boundaries and single films. - Figure 2 is the calculated Fresnel reflection coefficient for $\mathrm{Ni}$ and $\mathrm{W}$ at $\lambda=1.54 \AA$ for the case that the boundaries are perfectly smooth $(\sigma=0)$ or have a roughness with $\sigma=4 \AA$. The curves for the rough boundaries are obtained by multiplication of the Fresnel reflection

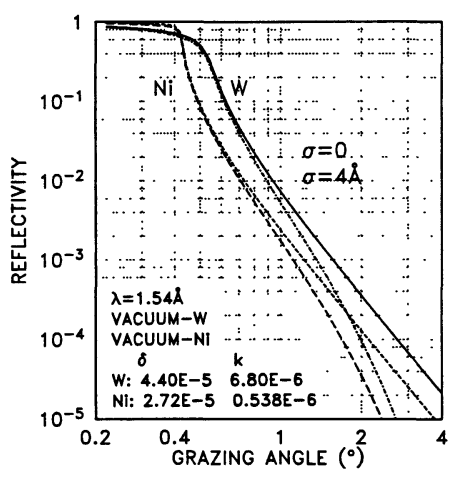

Fig. 2. - Calculated grazing incidence reflectivity for $\mathrm{Ni}$ and $\mathrm{W}$ for smooth boundaries and for a surface roughness of $4 \AA$.

with equation (18). From measurements of the reflectivity one can obtain $\delta, k$ and $\sigma$. One can rewrite the Fresnel equation by introducing a critical angle for total reflection

$$
\begin{gathered}
\cos \theta_{c}=\sin \alpha_{c}=\tilde{n}, \\
\sin \theta_{c}=\sqrt{2 \delta},
\end{gathered}
$$

which for materials with $k \ll \delta$ represents the angle, where the reflectivity drops from $R \simeq 1$ to very small values. For grazing angles $\theta_{0} \gg \theta_{\mathrm{cr}}$ the Fresnel formulas can be approximated by

$$
R=\frac{\left|\sin ^{2} \theta_{\mathrm{c} 2}-\sin ^{2} \theta_{\mathrm{c} 1}\right|^{2}}{16 \sin ^{4} \theta_{0}} .
$$

The reflectivity drops proportional to $1 / \sin ^{4} \theta_{0}$ in this range and one can obtain the critical angle or refractive index by extrapolating the straight lines in 
a $\log -\log$ plot as in figure 2 back to a reflectivity value of $1 / 16$. The absorption can easily be obtained by plotting the reflectivity as a function of $\theta_{0} / \theta_{\mathrm{cr}}$. All materials with the same ratio $\delta / k$ produce the same reflectivity curve and more gradual drops in the reflectivity are obtained for smaller values of $\delta / k$. By overlaying the measured curves to a set of reflectivity curves for different ratios $\delta / k$ that ratio can be directly read from such a plot $[6,7]$. Roughness of the boundary manifests itself in a drop of the reflectivity that is faster than $1 / \sin ^{4} \theta_{0}$ at larger angles. The measurement of small values of $\sigma$ requires reflectivity measurements at large critical angles, where reflectivities are low. Measurements with a conventional $\mathrm{Cu}-\mathrm{K} \alpha$ anode can measure reflectivities as low as $10^{-6}$ and can determine surface roughnesses around $1-2 \AA$. With synchrotron radiation [8] reflectivities in the $10^{-8}$ range have been measured.

In practice surfaces are often covered by a surface layer with different optical constants. For the case that this surface layer can be represented by a gradual transition of the optical constants from the bulk to vacuum, the surface can be described by a Debye-Waller type factor and its widths $\sigma$ is obtained in the same way as the roughness; roughness and a gradual transition layer cannot be distinguished in a measurement of the specular reflectivity. For the case that the surface is better described by a homogeneous film, oscillations produces by interferences between the top and bottom boundaries of the film will show up in the reflectivity curve. Figure 3

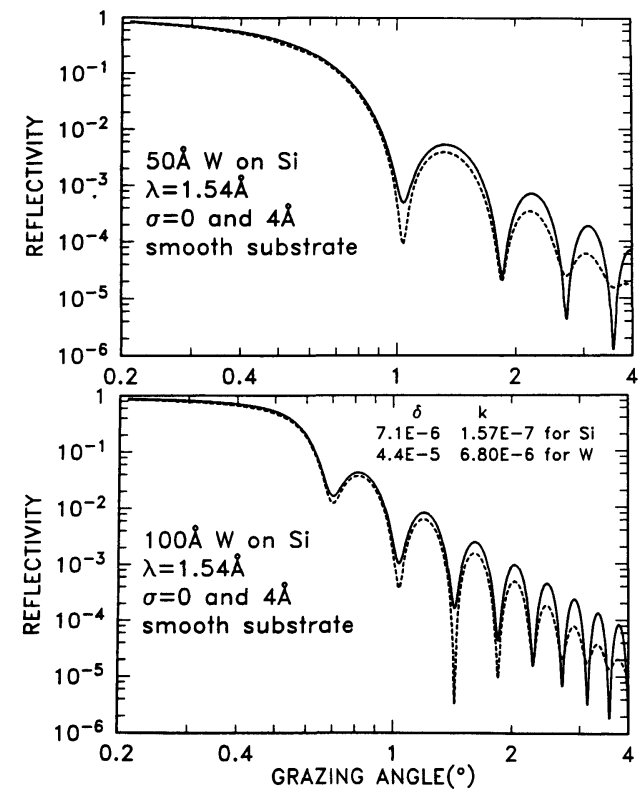

Fig. 3. - Calculated reflectivity versus grazing angle of incidence for a $50 \AA$ and $100 \AA$ thick W film on a Si substrate. Full curves are for smooth boundaries, the dashed curves for a roughness of $\sigma=4 \AA$ for the top surface. shows two examples for such interferences fringes. From measured data as in Figure 3 one can determine the optical constants of substrate and film and the roughness of the two boundaries. For angles larger than the critical angle, the reflectivity can be described by the vector model, i.e. the reflection corresponds to the diffraction pattern of two very narrow slits. From equation (11) we obtain the reflected amplitude

$$
r=r_{0}+r_{1} \mathrm{e}^{i q d},
$$

where $r_{0}$ and $r_{1}$ are the reflected amplitudes of the top and bottom boundary and $d$ is the film thickness. Equation (21) can be visualized in the complex plane as a rotation of the vector $r_{1}$ around the vector $r_{0}$ for the case that $r_{0}>r_{1}$, or as a rotation of the vector $r_{0}$ around $r_{1}$ if the contribution from the bottom boundary $r_{1}$ is larger [9]. Absorption in the film is described by the imaginary part of $q$. The period of the oscillation in real $q$-space $\Delta q$ or in angle of incidence is given by

$$
\Delta q d=2 \pi
$$

Films with larger thickness produce faster oscillations and require better collimation and monochromatization of the incident beam and higher precision in the goniometer. Because the reflectivity of a perfect interface decreases proportional to $1 / \sin ^{4} \theta_{0}$, for all materials, the average value and the amplitude of the oscillation decrease with the same function. Rough boundaries can be recognized by a faster drop. The contrast of the oscillation is largest $(100 \%)$, when both boundaries contribute with the same weight to the reflectivity $\left(\left|r_{0}\right|^{2}=\left|r_{1}\right|^{2}\right.$ in equation (21)) and decreases when the contributions become unbalanced. In figure 3 the top boundary has a higher Fresnel reflection coefficient ; in addition, the contribution of the bottom boundary is attenuated by absorption. The absorption decreases for steeper incidence angles which results in an increase of the contrast of the oscillation for increasing grazing angles for the case that the boundaries are smooth or have the same roughness. For boundaries with different roughness the relative contribution of the boundaries changes differently with increasing grazing angle. The dashed curves in figure 3 represent a film with a top boundary of $\sigma=4 \AA$ and a smooth bottom. For small grazing angles the contribution from the top is larger and the contrast in the oscillation is low. However, due to the roughness the contribution from the top decreases faster than that from the bottom. The effective contributions from both boundaries are equal at $\theta=1.5^{\circ}$ in figure $3 b$ and we observe a maximum contrast in the oscillation for this angle. For larger angles the relative contribution from the 
top decreases very fast. We observe an oscillation around the contribution from the bottom with an amplitude that decreases for increasing angles. A maximum in the contrast of the oscillation at some grazing angle for a high density film is always a sign for larger roughness of the top boundary. For the largest grazing angles only the reflectivity from the smoothest boundary remains visible.

The oscillations in figure 3 are equidistant in the variable $q$ or $\sin \theta$, where $\theta$ is the propagation angle inside the film. Due to refraction the oscillations are not equidistant in $\sin \theta_{0}$, the incident grazing angle in vacuum. We can use this fact to determine the refractive index as well as the thickness of the film from the reflectivity oscillations. Let us assume we are at a grazing angle $\theta \gg \theta_{\mathrm{cr}}$ and at a short wavelength were $k \ll \delta$. In this case the phase shifts on reflection are close to $0^{\circ}$ or $180^{\circ}$ for a transition to a material with a lower or higher electron density. For

$$
q d=2 \pi m, m=1,2,3 \ldots
$$

we have a maximum in the reflectivity when $r_{0}$ and $r_{1}$ are in phase (for a film with lower density than the substrate) and a minimum when they are shifted $180^{\circ}$ (for a film with higher density than the substrate). Choosing two extrema of order $m$ and $m^{\prime}$, we obtain 2 equations for the 2 variables $\delta$ and $d$. With equation (9) and Snell's law (Eq. (2)) we obtain for the index $\delta$ and the thickness $d$

$$
\begin{gathered}
2 \delta=\frac{\frac{\sin ^{2} \theta_{m}}{m^{2}}-\frac{\sin ^{2} \theta_{m^{\prime}}}{m^{\prime 2}}}{\frac{1}{m^{2}}-\frac{1}{m^{\prime 2}}}, \\
d=\frac{m \lambda}{2 \sin \theta_{m}} \frac{1}{\sqrt{1-\frac{2 \delta}{\sin ^{2} \theta_{m}}}},
\end{gathered}
$$

where $\theta_{m}$ and $\theta_{m^{\prime}}$ are the grazing angles for the two extrema of order $m$ and $m^{\prime}$ in vacuum. Minima should be used for a film of higher density than the substrate and maxima for a film of lower density. Equations (24) and (25) have already been used by Kiessig [10], and additional references to early workers are found in reference [11].

Henke et al. [12] have used a graphical method to solve equation (25) for $\delta$ and $d$. Using the Bragg condition $\quad d_{\mathrm{Bragg}}=\frac{m \lambda}{2 \sin \theta_{m}}$ and expanding the square root in equation (25) for $2 \delta \ll \sin ^{2} \theta_{m}$ one

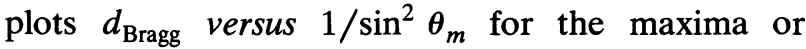
minima. The points should fall on a straight line that intersects the $y$-axis at $d$ and has the slope $2 \delta d$. One can avoid the approximation of the square root and use also extrema at very small grazing angles close to the critical angle if one plots $d_{\text {Bragg }}^{2}$ versus $1 / \sin ^{2} \theta_{m}$.
The points fall on a straight line that intersects the $y$ axis at $d^{2}$ and has the slope $2 \delta d^{2}$.

Multiple films. - Additional films produce additional oscillation periods in the $r(\theta)$ curve. We find 3 periods for a 2-layer system corresponding to the thicknesses of the 2 films and the total thickness of the structure. We still might be able to recognize the periods in a measured curve and derive the thicknesses and indices of the films from equations (24) and (25). The shortest period would give the total thickness of the structure and an effective index, which is the weighted average for the films. The reflectivity curve for a structure with $N$ different films is a superposition of $\frac{N}{2}(N+1)$ periods and one can usually not recognize all the periods directly from a look at the reflectivity curve of a structure with more than 2 films.

The parameters of such multilayer structures can be obtained by using a numerical minimization of a properly defined merit function (method (2) in Tab. I). Segmüller [13] defines a merit function

$$
F=\sum \frac{\left(R_{\exp }-R_{\mathrm{cal}}\right)^{2}}{R_{\exp }^{2}},
$$

where $R_{\text {exp }}$ and $R_{\text {cal }}$ are the measured and calculated reflectivities and the summation contains the data points. He uses the simplex method [14] to modify the coating parameters until a minimum in the merit function is reached. Figure 4 gives the result of such a minimization for a 11-layer coating of ReW-C [15].

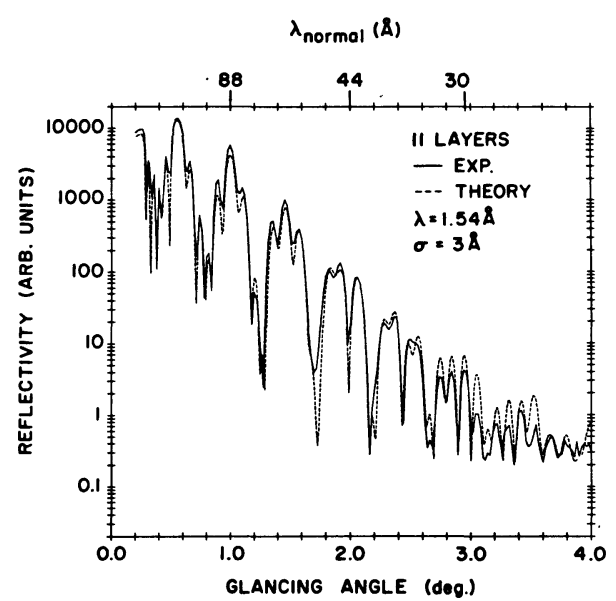

Fig. 4. - Measured (full curve) and calculated (dashed curve) reflectivity versus grazing angle for an 11-layer coating of ReW and C. Thicknesses for the calculated curve starting with $\mathrm{ReW}$ at the substrate : $22,70.4,17.7$, $69.8,15.6,76.1,13.4,87,12.5,80.9,9.8 \AA$; refractive indices of ReW and $\mathrm{C}: 1-4.4 \times 10^{-5}+i 6.8 \times 10^{-6}$ and $1-6.8 \times 10^{-6}+i 0.11 \times 10^{-6}$. The calculated reflectivity is multiplied with a Debye-Waller factor (Eq. (18)) with $\sigma=3 \AA$ (from Ref. [15]). 
The required computer time increases rapidly for an increasing number of layers. Furthermore the solution is not unique and might converge to a wrong structure. For this reason we usually do not use this method for coatings which contain more than about 10 layers.

DIRECT METHODS, INVERSION BY FOURIER TRANSFORM. - Direct inversion methods are plagued by two difficulties : first, the mathematics for a general inversion is cumbersome and second, and more important, the phases are unknown. Direct inversion methods have been used in the design of coatings for visible light [16], and Skita et al. [17] have used direct methods to determine the density profile within Langmuir-Blodgett films. In the approximation of the vector model, the mathematical inversion becomes trivial. The reflectivity as a function of depth is simply obtained by an inverse Fourier
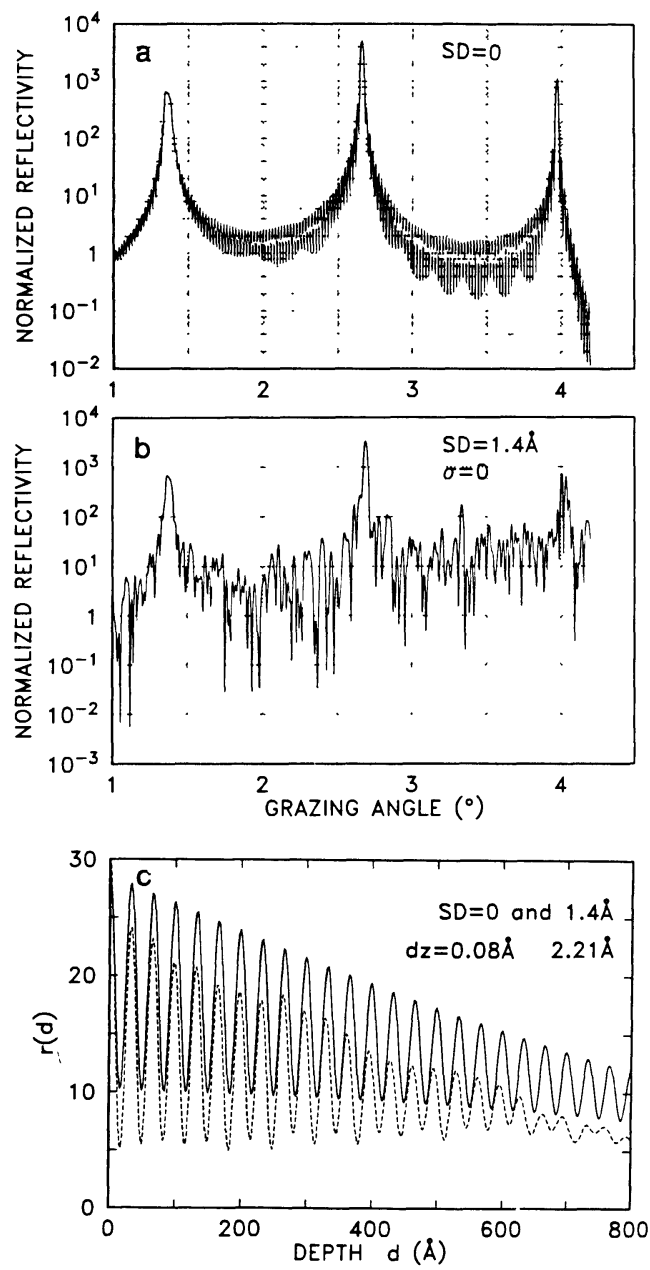

Fig. 5. - (a) Reflectivity normalized to the reflectivity of a single boundary for the 128 layer periodic coating as in figure 1 ; (b) for a 128 layer coating with compensated thickness errors with standard deviation $\mathrm{SD}=1.4 \AA$; (c) Inverse Fourier transform of the curves in (a) and (b) ; $\mathrm{d} z$ is the standard deviation in the distance between subsequent maxima. transformation of the reflected amplitude $r(q)$ in equation (14). However, measurements give only the intensity $I=r(q) \cdot r^{*}(q)$ over a limited range of $q$ values. A Fourier transformation of the measured intensity gives the weight with which different distances in the structure contribute to the reflectivity, but not the structure itself. Figure 5 gives an example. The input were the calculated $R(q)$ curves for the periodic multilayer of figure 1 and that for the same coating with a compensated thickness error with a standard deviation $\Delta d=1.4 \AA$. We normalize this reflectivity to the single boundary reflectivity and use $R_{12}=\left(\delta_{1}-\delta_{2}\right) / 4 \sin ^{4} \theta$ as approximation. The inverse Fourier transform yields an oscillating structure with a constant period for the error-free coating and a more irregular pattern for the coating with errors. The errors in the position of the maxima of Figure 1c are larger than the thickness errors in the original coating. For still larger errors, the inverse Fourier transform yields a very irregular, speckle like pattern, which is difficult to interpret. The method has its analog in X-ray diffraction and the resulting distributions are called Patterson patterns [18].

In the plots in figure 1 the oscillations in the phase are locked to the oscillations in the reflected intensity. This suggests that the phase function is not arbitrary, but can be derived from the measured reflectivity. Causality arguments can be used to derive a dispersion relation between amplitude and phase [19]. Furthermore, the phase function has to be chosen in such a way that $r(z)=0$ outside the multilayer coating [17]. In principle, one should be able to use these methods to obtain an unique reconstruction of the multilayer. In practice, we do not know at the present time if direct methods have advantages over the modeling techniques.

STATISTICAL CHARACTERIZATION OF A MULTILAYER. - For nearly periodic multilayer coatings with many layers, the number of free parameters can be drastically reduced by using a statistical approach. Instead of determining the thickness of each individual layer, we determine only the average deviation of the thickness from its nominal value. A coating is then described by only a few parameters: the nominal period $p$, the thickness ratio of the two materials, the standard deviation $\Delta \mathrm{d}$ of the thicknesses from their nominal values, and the roughness $\sigma$ of the interfaces. Most important for the performance of a multilayer are the thickness errors and the roughness of the boundaries. Both reduce the maximum reflectivity of a mirror. It is important for improvements in the fabrication process to know which of these two parameters is bigger and we will show in this section how one can obtain these parameters separately from a measured reflectivity curve. 
The influence of thickness errors and roughness on the performance of an X-ray mirror is demonstrated in the model calculations of figure 6 , which should be compared to the reflectivity of the perfect mirror in figure 1 . We recognize the following behaviour :

(1) both, thickness errors and roughness reduce the peak reflectivities and the higher order maxima are more strongly affected than the first order peak ;

(2) increasing thickness errors reduce the reflectivity of the main maxima to values close to $N R_{12}$;

(3) thickness errors increase the reflectivities between the peaks, especially at the $1 / 2,3 / 2,5 / 2$ order, and the reflectivity approaches the value $N R_{12}$ as thickness errors increase ;

(4) roughness decreases the reflectivity at the maxima and also between the peaks. For large grazing angles the reflectivity decreases faster than $\propto \sin ^{4} \theta$ for coatings with rough boundaries ;

(5) the regular spacings between the side maximum becomes irregular when thickness errors are introduced.

The period and the effective refractive index of a periodic multilayer can be obtained from the angle of the main maxima using equations (24) and (25) where $m$ is now the order of a reflection and $d$ becomes the period of the multilayer. The influence
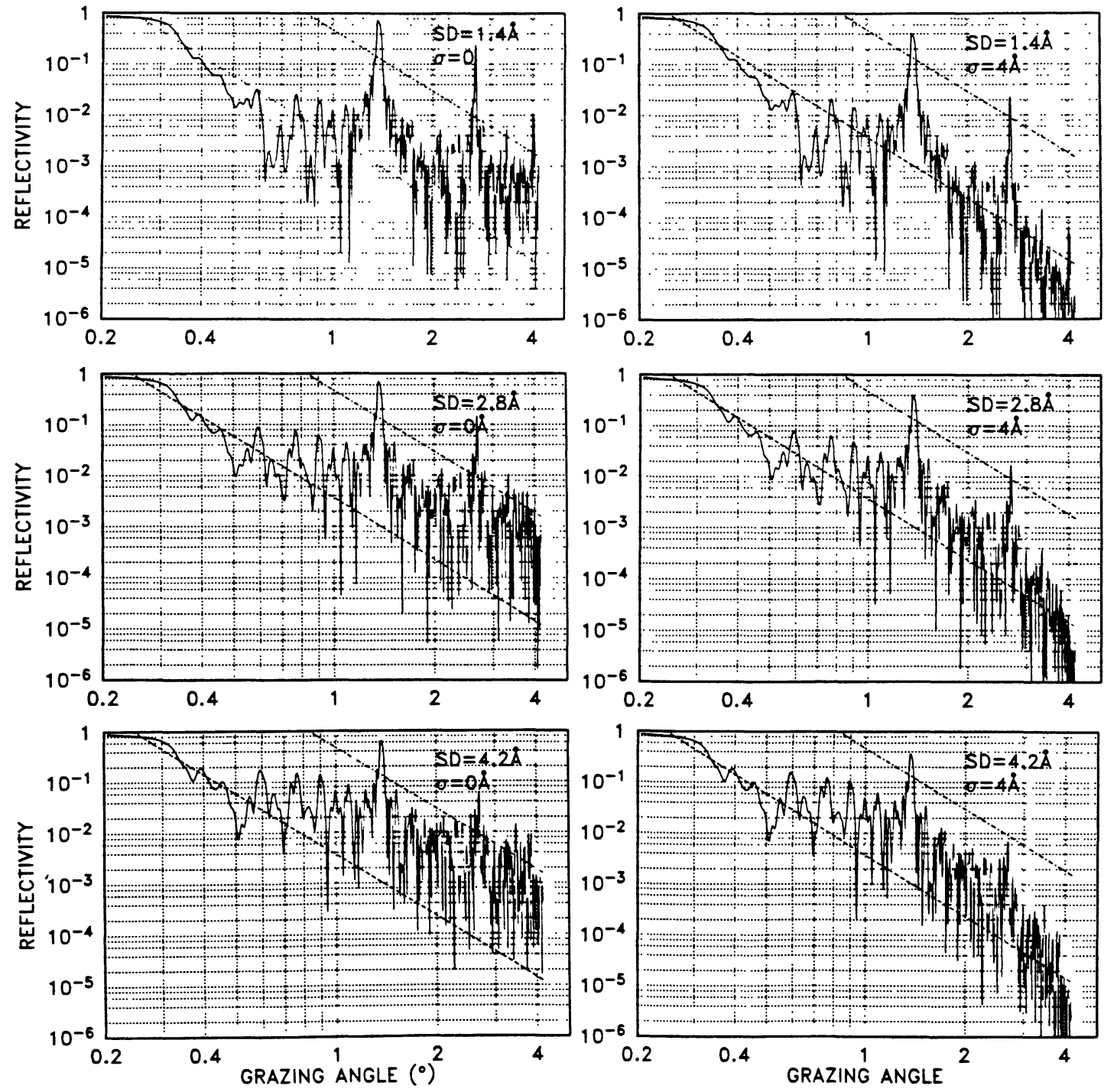

Fig. 6. - Calculated reflectivities at $\lambda=1.54 \AA$ for the 128 layer $\mathrm{X}$-ray mirrors of figure 1 with compensated thickness errors with a standard deviation $\mathrm{SD}=1.4,2.8$ and $4.2 \AA$ as they might occur with in situ monitoring of the reflectivity during deposition, both for smooth boundaries (left), and boundaries with $4 \AA$ roughness (right). The error compensation is simulated by multiplying the errors obtained by a random walk process with a Gaussian to reduce the maximum error and by changing the sign of a component of the error as soon as the accumulated error exceeds a maximum value. The dashed curves represent $R_{12}$ and $128 R_{12}$, where $R_{12}$ is the reflectivity of a single boundary. 
of both imperfections, roughness and thickness errors, on the reflectivity is largest at the largest grazing angles, where the vector model gives a good description of the reflectivity. In this model the multilayer is equivalent to a diffraction grating with very small openings. For a perfectly periodic grating of $N$ narrow slits the diffracted amplitudes add in phase at the maxima. The diffracted intensity at a maximum is $N^{2}$ times the intensity from a single opening. The coating with very large thickness errors is equivalent to a diffracting screen where the openings are located at random position and all openings add their amplitudes with random phases. In this case the intensities from all opening are added and we expect $\mathrm{N}$ times the intensity from a single opening.

No temporal phase fluctuations occur in a coating and the reflectivity curve is equivalent to a diffraction pattern in coherent light. Such patterns contain large oscillations with contrast 1 around the expectation value. The superposition of many waves with pseudo-random phases generates a speckle pattern, where the probability density for the intensity $I$ is given by [20] :

$$
p(I)=\frac{1}{\langle I\rangle} \exp -\frac{I}{\langle I\rangle},
$$

and $\langle I\rangle$ is the expectation value of the intensity $I$. For a specific coating at a specific grazing angle the reflectivity can differ considerably from its expectation value (see Fig. 6).

The expectation value for the reflectivity $\langle R\rangle$ at a maximum of order $m$ for non-accumulating thickness errors is given by [4]

$$
\begin{aligned}
\langle R\rangle= & N R_{12}\left(1-\exp \left(-4\left\langle\Delta \varphi^{2}\right\rangle\right)\right) \\
& +\sin ^{2} \gamma N^{2} R_{12} \exp \left(-4\left\langle\Delta \varphi^{2}\right\rangle\right),
\end{aligned}
$$

for

$$
m=2,3,4 \ldots
$$

where $R_{12}$ is the reflectivity from a single boundary (or the intensity from one opening in the grating) and $\left\langle\Delta \varphi^{2}\right\rangle$ is the variance in the phase $\varphi$ due to random thickness errors (Eq. (11)). $\gamma=m \pi d_{1} / p$ describes the nominal phase separation between the top and bottom of film 1 of thickness $d_{1}$ within one period. We have $\sin \gamma=1$ for the quarter wave stack and $m=1$, where $d_{1}=d_{2}=p / 2$, with $p=\frac{\lambda_{\max }}{2 n \cos \alpha_{\max }}$ being the period in the multilayer. The value of $\sin \gamma$ is close to 1 for practical X-ray mirrors at the first order peak $(\sin \gamma=0.87$ for $d_{1}=p / 3$ ). For a periodic multilayer without thickness errors the contributions from the top and bottom of each film cancel for $m=p / d_{1}$ and we have $\sin \gamma=0$. In figures 1 and 5a we recognize this condition as very low reflectivity at $m=3.345$ above the 3rd-order maximum. However, this minimum usually does not appear in real coatings with thickness errors (see Fig. 5b) or in coatings where $d_{1} / p$ changes throughout a coating. Replacing $\sin ^{2} \gamma$ by its average value $\sin ^{2} \gamma=0.5$ is often a good approximation to describe the reflectivity at large grazing angles and we will use this value for our examples. Exceptions are coatings with small thickness errors, especially those designed to suppress a specific order where $p / d_{1}$ is a small integer. The minimum of $\sin \gamma$ is recognized by inspection of the reflectivity curve for these cases and the proper value of $\sin ^{2} \gamma$ should be used for each grazing angle.

The first term in equation (28a) is zero for a coating without thickness errors and approaches the value $N R_{12}$ for large errors, describing the addition of the reflected intensities from all boundaries. The second term describes the addition of the amplitudes from each period with equal phases for small thickness errors and approaches zero for large errors.

At a half order $(m=1.5,2.5,3.5 \ldots)$ four adjacent boundaries add up to zero for an error free coating and the reflectivity oscillates around $R_{12}$ in the neighbourhood of the minimum. We replace the second term in equation (28a) with the value $R_{12}$ and obtain an expression which gives us the envelope through the reflection near half orders

$$
\langle R\rangle=N R_{12}\left(1-\exp \left(-4\left\langle\Delta \varphi^{2}\right\rangle\right)\right)+R_{12},
$$

for

$$
m=1.5,2.5,3.5 \ldots
$$

Figure 7 is a plot of equations (28a) and (28b). The curves demonstrate the high sensitivity of the reflectivity near half-orders towards small thickness errors.

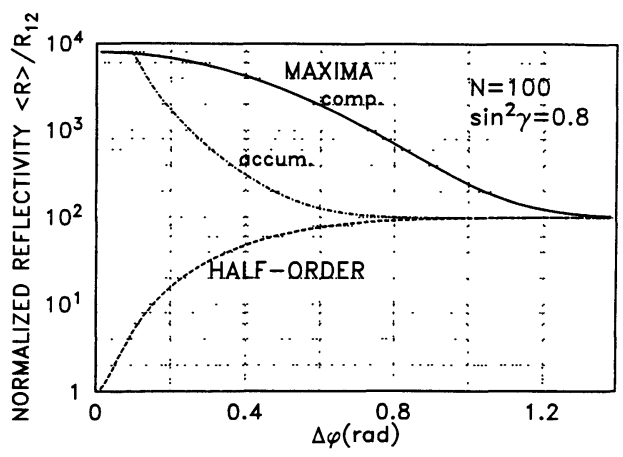

Fig. 7. - Expectation value of the reflectivity normalized to the reflectivity of a single boundary versus the phase error caused by thickness errors in the layers for the maxima at integer orders for compensated (full curve) and accumulating (dashed curve) errors and for the minima around half-orders obtained from equations (28) and (36). The discontinuity in the slope of the curve for accumulating errors occurs where $N_{\text {coh }}$ is replaced by $N$ for $N_{\text {coh }}>N$. The bottom scale is proportional to $\sin \theta$ for a specific coating (see Eq. (5a)). 
The ratio between the maxima and minima $\Gamma$ in the reflectivity curve (the ratio between the top and bottom curves in Fig. 7) can be used to obtain the variance $\left\langle\Delta \varphi^{2}\right\rangle$ :

$$
\left\langle\Delta \varphi^{2}\right\rangle=\frac{1}{4} \ln \frac{N \sin ^{2} \gamma+\Gamma-1}{\Gamma+\Gamma / N-1} .
$$

Equation (29) gives the thickness errors in a coating from a simple inspection of the measured reflectivity curve in the range where the vector model is valid. For an error-free coating the maxima are a factor $\sin ^{2} \gamma N^{2}$ higher than the surrounding minima and the decrease of the ratio is a measure for the errors. The ratios obtained are the same for rough and smooth boundaries, i.e. the method gives the thickness error independent of roughness. We can express the error in $\varphi$ by a thickness error (Eq. (5)),

$$
\Delta d=(p / m \pi) \Delta \varphi,
$$

where $\Delta d$ and $\Delta \varphi$ denote the standard deviation, $\Delta \varphi=\sqrt{\left\langle\Delta \varphi^{2}\right\rangle}$, and $m$ is the order of the reflection.

Roughness can now be obtained by comparing the measured reflectivity curve to its calculated value, where thickness errors are included in the calculation. The simplest way to do this is by comparison at very large grazing angle, where the measured value of the reflectivity approaches $N R_{12} \exp (-q \sigma)^{2}$. At half orders equations (18) and $(28 \mathrm{~b})$ give the expectation value of the reflectivity of a mirror with roughness and thickness errors as

$$
\begin{aligned}
\langle R\rangle=N R_{12} \exp \left(-q_{m}^{2}\right. & \left.\sigma^{2}\right) \times \\
& \times\left(1-\exp \left(-4\left\langle\Delta \varphi^{2}\right\rangle\right)\right) \\
& +R_{12} \exp \left(-q_{m}^{2} \sigma^{2}\right),
\end{aligned}
$$

which can be solved for $\sigma$ :

$$
\begin{aligned}
\mathrm{e}^{q_{m}^{2} \sigma^{2}}= & \frac{R_{12}}{\langle R\rangle} \\
& {\left[1+N\left(1-\exp \left(-\frac{4 \pi^{2} m^{2}}{p^{2}}\left\langle\Delta d^{2}\right\rangle\right)\right)\right] }
\end{aligned}
$$

for

$$
\begin{gathered}
m=1.5,2.5,3.5 \ldots \\
q_{m}=2 \pi m / p
\end{gathered}
$$

The value of $R_{12}$ as calculated from the optical constants is needed to derive the roughness from equation (32). By measuring the reflectivity at 2 adjacent half-orders one obtains 2 equations for the variables $\sigma$ and $\left\langle\Delta \varphi^{2}\right\rangle$. Only ratios between $R_{12}$ values occur in the solution and these can be eliminated by using the fact that $R_{12} \propto 1 / \mathrm{m}^{4}$. We obtain the roughness from

$$
\begin{aligned}
& \mathrm{e}^{\left(q_{m+1}^{2}-q_{m}^{2}\right) \sigma^{2}}=\frac{\left\langle R_{m}\right\rangle}{\left\langle R_{m+1}\right\rangle} \frac{m^{4}}{(m+1)^{4}} \\
& \frac{1+N\left[1-\exp \left(-\frac{4 \pi^{2}(m+1)^{2}}{p^{2}}\left\langle\Delta d^{2}\right\rangle\right)\right]}{1+N\left[1-\exp \left(-\frac{4 \pi^{2} m^{2}}{p^{2}}\left\langle\Delta d^{2}\right\rangle\right)\right]},
\end{aligned}
$$

for

$$
m=1.5,2.5,3.5 \ldots
$$

where $\left\langle R_{m}\right\rangle$ is the measured reflectivity at half order $m$.

Accumulating errors. - Equations (28)-(33) are derived for the case that the positions of all boundaries on average have the same error, i.e. thickness errors are not accumulating as is realized during deposition with in situ monitoring of the reflectivity. For deposition without in situ control, the thickness errors in one layer can add to the errors in the layers beneath, and errors can accumulate to considerably larger values than the error for the individual layer. At half-order locations in the reflectivity curve, where 4 adjacent boundaries add to zero for the error-free coating, there will be little difference between compensated and accumulating errors. We can use the same statistical treatment for both cases, and we can apply equation (31) to 2 half-orders to give us 2 equations to determine both roughness and thickness errors. If we use the ratio $\Gamma$ in equation (29) to determine thickness errors, we overestimate the error and we call the value obtained the equivalent error $\Delta d_{\text {equiv }}$ for the compensating case. For random accumulation of errors we expect that the accumulated error after the addition of $N$ films is given by

$$
\Delta d_{\mathrm{acc}}=\Delta d \sqrt{N}
$$

We now assume that we only have in-phase addition of the reflected amplitudes over subgroups of films which have sufficiently small error accumulation, and we add intensities when we add the contributions from the different subgroups in a coating. Defining the number of layers $N_{\text {coh }}$ in such a subgroup by the condition that the accumulated phase error is smaller than $180^{\circ}$,

$$
N_{\text {coh }}=\frac{1}{\left\langle\Delta \varphi^{2}\right\rangle},
$$

we obtain for the expected reflectivity at a maximum :

$$
\begin{aligned}
\langle R\rangle= & N R_{12}\left(1-\exp \left(-4\left\langle\Delta \varphi^{2}\right\rangle\right)\right)+ \\
& +\sin ^{2} \gamma N N_{\operatorname{coh}} R_{12} \exp \left(-4\left\langle\Delta \varphi^{2}\right\rangle\right),
\end{aligned}
$$

with $N_{\text {coh }} \leq N$. Equation (36) is plotted as dashed curve in figure 7 . For very small errors, where all 
boundaries still add amplitudes in phase, and for very large errors, where intensity are added in both cases, accumulating and compensating errors give the same peak reflectivity. For intermediate errors we obtain a lower peak reflectivity for the case of accumulating errors. We should note that equation (36) gives the expectation value of the reflectivity and we can expect to measure that value if we average over a large number of coatings. An individual coating may contain only few subgroups and the actual reflectivity may differ considerably from the statistically predicted expectation value.

COATINGS OPTIMIZED FOR ROUGHNESS ANALYSIS. - The highest sensitivity to roughness is obtained for the largest values of $q$ or the largest grazing angles of incidence. However, reflectivities are very low in this region and the dynamical range in the reflectivity measurements limits the possible $q$ range. For a perfectly error-free periodic coating with $N$ layers, the peak reflectivity is close to $N^{2} R_{12}$. A coating with 100 layers can have a $10^{4}$ times larger peak reflectivity than the single boundary and therefore the peak reflectivities can be measured for considerably larger $q$ values. However, we have to compare the peak reflectivity to the reflectivity between integer orders to confirm that the coating can be considered as error-free and this requires measured reflectivities of the order of the single boundary reflectivity. For the case that this reflectivity is below the background in a measurement, we can estimate only the combined influence of roughness and thickness errors on the reflectivity of the high order maxima and can not derive both parameters independently.

A higher sensitivity for the characterization of the boundaries can be obtained if one uses multilayers with many layers and large thickness errors. Thickness errors should be sufficiently large that for all $q$ values of interest the second term in equation (28) is small compared to the first. In this case the expectation value for the reflectivity is $N R_{12}$ for all sufficiently large $q$ values. We can plot the ratio $\langle R\rangle / N R_{12}(\langle R\rangle$ is the measured expectation value of the reflectivity) as a generalized Debye-Waller function. An analysis of this function can give us by Fourier transform the actual transition function at a boundary. In many coatings boundaries have different roughness (in a metal-carbon multilayer the boundary from carbon to metal is smoother than that from metal to carbon) and one can try to deduce both values by decomposing the measured curve into a superposition of two Debye-Waller factors with different $\sigma$ values. The roughness of the boundaries may change from the bottom to the top of a multilayer coating. For large grazing angles all boundaries contribute with equal weight to the reflected amplitude and a change in roughness throughout the stack can not be recognized from the reflectivity. However, the penetration depth into a coating decreases with decreasing grazing angle. Therefore the reflectivity at small grazing angles is more sensitive to the top of the coating and the analysis in this range will produce roughness values that correspond more to the top of the coating. A roughness that is a function of the grazing angle can be seen as a sign for a change in the roughness throughout the coating. One problem with this analysis is that roughness affects the reflectivity very little at small grazing angles. It is usually better to analyse the reflectivity in situ during the deposition in order to observe a change in roughness throughout the coating $[9,21]$.

EXAMPLES. - Figures 8 and 9 are measured reflectivity curves for mirror coatings and we will use these curves as examples for our statistical analysis. We first determine the period and the effective refractive index from the position of the maxima using equations (24) and (25) and obtain values of $p=72.2 \AA$ and $\delta_{\text {eff }}=1.4 \times 10^{-5}$ for figure 8 and values of

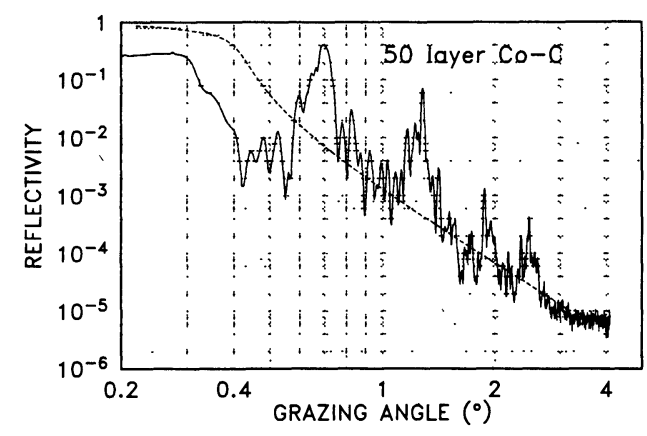

Fig. 8. - Measured reflectivity at $\lambda=1.54 \AA$ of a 50 -layer Co-C multilayer with a period $p=72.2 \AA$ (full curve). The dashed curve is the calculated reflectivity $R_{12}$ for a smooth internal boundary. The coating was fabricated with in situ reflectivity monitoring and contains compensated thickness errors.

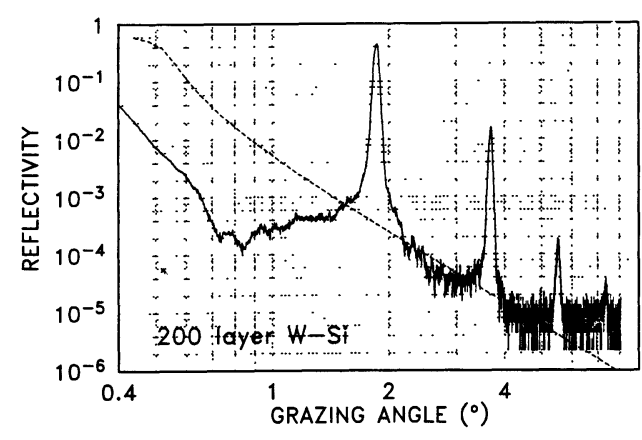

Fig. 9. - Measured reflectivity at $\lambda=1.54 \AA$ of a commercial 200-layer W-Si multilayer with a period $p=24.1 \AA$. The dashed curve represents the reflectivity of a single internal boundary $R_{12}$. 
$p=24.1 \AA$ and $\delta_{\text {eff }}=1.7 \times 10^{-5}$ for figure 9. From the irregularity of the structure between the maxima and the fact that the reflectivity at order $m=1.5$ is above the single boundary value in figure 8 we can see that thickness errors are present. The fact that the envelope through the half-order points decreases faster than the single boundary reflectivity indicates roughness. We read the ratio $\Gamma$ between the reflectivity maxima and the envelope through the halforder reflectivity as $\Gamma=60,18,10$ for $m=2,3,4$ and use equation (29) with $\sin ^{2} \gamma=0.5$ to obtain the standard deviation of the thicknesses $\Delta d$. We obtain an average value $\Delta d=3.4 \AA$. From the design of the coating we would expect a value $\sin \gamma \simeq 0$ at the $3^{\text {rd }}$ maximum. However, inspection of the reflectivity curve shows very little reduction in $R$ around $m=3$ and therefore we use the average value 0.5 of $\sin ^{2} \gamma$ for all grazing angles. We derive the roughness of the boundaries from equation (32) using the values of $R_{12}$ and $\langle R\rangle$ read from figure 8 and a thickness error $\Delta d=3.4 \AA$. The results of the analysis are tabulated in table II. Note that we obtain larger roughness values at smaller values of $m$. Because the top boundaries contribute with larger relative weight at smaller grazing angles, we conclude that the roughness for the coating in figure 8 increases from the bottom to the top of the coating. This observation is in agreement with the results of Brujn [21] obtained from monitoring the reflectivity during film deposition and with tests of the coating near normal incidence with soft X-rays.

Table II. - Characterization of the 50 layer coating in figure 8. Period $p=72.2 \AA, \quad \delta_{\text {eff }}=1.4 \times 10^{-5}$. Thickness errors $\Delta d$ are obtained from equation (29), roughness $\sigma$ from equation (32) at order $m$.

\begin{tabular}{|lcc|}
\hline$m$ & $\Delta d(\AA)$ & $\sigma(\AA)$ \\
\hline 1.5 & & 8.4 \\
2 & 3.3 & \\
2.5 & & 7.4 \\
3 & 3.6 & \\
3.5 & & 5.7 \\
4 & 3.3 & \\
4.5 & & 4.9 \\
\hline
\end{tabular}

We can use our result to estimate the performance of the mirror in first order reflectivity. Assuming a Debye-Waller type factor for both the roughness and thickness errors we find a value of 0.89 due to thickness errors and a factor of 0.6 due to an average roughness of $7.5 \AA$. The total expected reduction in reflectivity would be a factor of 0.58 . The actual reduction might be still smaller due to the fact that thickness errors tend to be smaller near the end of the deposition, when the deposition system has stabilized. Furthermore, the full theory for high reflectivity (where the vector model no longer applies) shows that multiplication of the peak reflectivity with a Debye-Waller factor overstates the drop in reflectivity [4].

The coating in figure 9 was fabricated without in situ monitoring of the reflectivity, and we expect accumulation of thickness errors. The reflectivity at half-order is below background for $m>2$. We have to extrapolate the half-order intensity from $m=1.5$ to $m=2.5$, if we want to derive the thickness errors. We estimate values $\Gamma=2000,200$ for $m=2$ and 3 and use equation (29) to obtain the equivalent thickness error for a non-accumulating coating $\Delta d_{\text {equiv }}=0.4$ and $0.8 \AA$. We can recognize from figure 9 that the performance of the coating is affected by roughness : at $m=1.5$ the measured reflectivity is below the reflectivity value of the single boundary. Inserting the measured reflectivity at $m=1.5$ into equation (32) with $\Delta d=0.5 \AA$ we obtain a value $\sigma=2.9 \AA$. We can also estimate the combined influence of thickness errors and roughness on the reflectivity by comparing the measured reflectivities for orders $m=2,3$ to their theoretical value $\sin ^{2} \gamma N^{2} R_{12}$ and fitting that ratio to a DebyeWaller factor. We obtain $\sigma_{\text {total }}=3.4 \AA$, a value in reasonable agreement with the values obtained for $\Delta d$ and $\sigma$. The corresponding Debye-Waller factor for the first order maximum would have a value of 0.46 . Using the roughness value $\sigma=2.9 \AA$ and the measured and calculated reflectivity for $m=1.5$ in figure 9 we obtain the standard deviation of the thickness errors from equation (31) as $\Delta d=0.2 \AA$. This leads to a phase error $\Delta \varphi=0.026$ at the first order peak and yields a value $N_{\text {coh }}=1400$, a value considerably larger than the actual number of layers in the coating. We conclude that thickness errors do not reduce the reflectivity of the first order maximum in figure 9.

\section{Summary.}

The structure of a multilayer mirror in the direction normal to the layers can be deduced from a measured reflectivity curve. For a reliable reconstruction the number of data points in the measurement should be considerably larger than the number of free parameters in the structure. Reflectivity measurements at short X-ray wavelengths are preferred over measurements in the soft $\mathrm{X}$-ray range, because optical constants are better known at shorter wavelengths, and usually measurements over a larger range in momentum space are obtainable. For structures with few layers a look at the reflectivity curve often provides a good guess about the structure, and this first guess can be refined with model calculations until satisfactory agreement is obtained. 
The refinement can be automated by defining a merit function, which describes the quality of the fit between experiment and model, and by minimizing this merit function numerically. The method works reliably up to about 10 layers in a coating and yields the thickness of each layer and the roughness of each interface. Direct inversion methods as used in X-ray diffraction have to estimate phases from the measured reflectivity and from a priori information and might become useful for the analysis of multilayer coatings. However, only limited experience with these methods is available today. For mirror coatings with a large number of layers a statistical description of the multilayer is appropriate. The average period, the standard deviation of the layer thicknesses from their nominal value, and the average roughness can be derived from a reflectivity curve and simple formulas. These methods are discussed for the case of compensated and of accumulating errors. The parameters obtained can be used in model calculations to predict the performance of the coating at any angle of incidence and wave-length, where optical constants are known. They also serve as a guide for changes in the fabrication process to produce better coatings.

\section{Acknowledgments.}

Alan E. Rosenbluth and Armin Segmüller have made numerous contributions to the field covered in this paper, and I thank them for many helpful discussions over many years on most of the topics covered. R. E. Somekh and the reviewer of this paper provided me with valuable comments which were used to improve the manuscript.

\section{References}

[1] Heavens, P. S., Optical Properties of Thin Films (Dover, New York) 1965 ;

VAsiceK, A., Optics of Thin Films (North Holland, Amsterdam) 1960 ;

MACleOD, H. A., Thin-Film Optical Filters, (Elsevier, New York) 1969 ;

Born, M. and Wolf, E., Principles of Optics, 5th edition (Pergamon Press) 1975 ;

Berning, P. H., Theory and Calculations of Optical Thin Films, in Physics of Thin Films, Ed. G. Hass (Academic Press, New York) 1 (1963) p. 69.

[2] Ewald, P. P., Ann. Phys. 49 (1916), 1, 117 ;

JAMES, R. W., The Optical Principles of Diffraction of X-rays (Cornell U. Press, Ithaca New York) 1965 ;

VON LAUE, M., Röntgenstrahl-Interferenzen (Akademischer Verlag, Frankfurt) 1960 ;

Batterman, B. W. and Cole, H., Rev. Mod. Phys. 36 (1964) 681.

[3] Henke, B. L., Lee, P., Tanaka, T. J., ShimabukURO, R. L. and FujIKAwA, B. K., AIP Proc. 75 (1981) 340 ; At. Data Nucl. Data Tables, 27 (1982) 1.

[4] Spiller, E. and Rosenbluth, Alan A., Proc. SPIE 563 (1985) 221-236 ; Opt. Eng. 25 (1986) 954963.

[5] Ed. H. P. Baltes, Inverse Source Problems in Optics, in Topics in Current Physics (Springer, Berlin) 1978 ;

BAtes, R. H. T. and McDonnell, M. J., Image restoration and reconstruction (Clarendon Press, Oxford) 1986.

[6] Compton and Allison, S. K., X-ray in Theory and Experiment (van Nostrand, New York) 1935.
[7] Parratt, L. G., Phys. Rev. 95 (1954) 359.

[8] Weiss, A. H., Deutsch, M., Braslau, A., Оско, B. M. and Pershan, P. S., Rev. Sci. Instrum. 57 (1986) 2554 ;

Braslau, A., Deutsch, M., Pershan, P. S., Weiss, A. H., Als-Nielsen, J. and Bohr, J., Phys. Rev. Lett. 54 (1986) 114.

[9] SPILler, E., Proc. SPIE 563 (1985) 367-375.

[10] Kiessig, H., Ann. Phys. 5.F 10 (1931) 769.

[11] Croce, P., Devant, G., Sere, M. G. and VerHAEGHE, M. F., Surf. Sci. 22 (1970) 173.

[12] Henke, B. L., Uejio, J. Y., Yamada, H. T. and TACKABERry, R. T., Opt. Eng. 25 (1986) 937.

[13] Segmüller, A., A.I.P. Conf. Proc. 53 (1979) 78.

[14] Nelder, J. A. and Mead, R., Comput. J. 7 (1965) 308.

[15] Haelbich, R.-P., Segmüller, A. and Spiller, E., Appl. Phys. Lett. 34 (1979) 184.

[16] Liddell, H. M., Computer-aide techniques for the design of multilayer filters (Adam Hilger Ltd, Bristol) 1981.

[17] Skita, V., Filipkowski, M., Garito, A. F. and Blasie, J. K., Phys. Rev. B 34 (1986) 5826.

[18] Patterson, A. L., Phys. Rev. 65 (1944) 195.

[19] Stern, F., Solid State Phys., Eds. F. Seitz and D. Turnbull (Academic Press, New York) 15 (1963) 328.

[20] DainTy, J. C., Laser Speckle and Related Phenomena, Top. Appl. Phys., 2nd edition (Springer Verlag, Berlin) 9 (1984).

[21] BRuiJn, M. P., Deposition and characterization of multilayer X-ray reflection coatings, Thesis, FOM-Institut Amsterdam (1986). 